\title{
Acoustic Location System based on the Cumulative Sum Algorithm
}

\author{
Xianhao Shen, He Nai \\ Xianhao Shen, Ph. D., Guilin University of Technology, NO.12 Jiangan Road, \\ Guilin, Guangxi Province, China. (541004) Tel:15077301839 \\ He Nai, postgraduate, Guilin University of Technology, NO.12 Jiangan Road, \\ Guilin, Guangxi Province, China. (541004) Tel:18290065616 \\ 25337698@qq.com,985515263@qq.com
}

\begin{abstract}
Before we apply the Time Difference of Arrival (TDOA) to locate sound target, we should have known arrival time of each sensor node at first, we present two algorithms based on the cumulative sum to estimate the arrival time. We analysis influences of location accuracy, caused by five sensors position algorithm and the distance of each node. Taking these several factors, we find the best distance between sound source and node and propose a node organizational method-retest measure method. According to the consequence of first measure, organize node to locate again. The result of simulation shows that these algorithms are in effect and the retest measure can reduce deviation effectively.
\end{abstract}

Keywords: Sound target; sensor; algorithm; location accuracy; retest measure method

\section{Introduction}

In the field of the Internet of Things, using wireless sensor network to achieve sound signal of target and localizing the sound source position further. It uses a passive detection method, so it has a strong concealment .Even if the measured target use the way of stealth and interference to hide itself, it will also issue a lot of noise. Wireless sensor networks can just use this feature to locate the target.

It already have three basic measure to locate sound target: Controllable beam forming technology based on maximum output power, High resolution spectral estimation technique and Positioning technology based on acoustic time difference(TDOA) ${ }^{[1]}$. Because of the technology of TDOA has low complexity and easy to implement, it was widely used. In the technology of TDOA, we should estimate the time of arrival. The known Generalized cross correlation function $(\mathrm{GCC})^{[2]}$ is by filtering the received signal, eliminate the influence of noise and interference, then take correlation calculation for maximum and estimate the arrival time. Signal through a finite impulse response filter, arrival time estimation will be converted into filter parameters estimation. By interpolation filter parameters in real time will be able to estimate arrival time. The above process is Least mean square adaptive filter (LMS) ${ }^{[3]}$. Another way is based on the cross correlative function which is the signal capture by two sensor node. Searching for the maximum value of cross-correlation function can found the difference of arrival time, this is Cross power spectrum phase method ${ }^{[4]}$. But because of these algorithm is complex, it need the node should have much computing resources, this made the cost high.

This paper presents two algorithms to estimate arrival time, these algorithm can effectively reduce the node costs because of the low complexity. And we also present an organizational method of nodes, reducing the system's position error. 


\section{Mathematical Model of Acoustic Events}

\subsection{Sound Model}

Consider the

$$
\begin{gathered}
\text { mathematical model } \\
\qquad x[n]= \begin{cases}v[n] & \text { of } \\
\operatorname{as}[n-\tau]+v[n] & n>\tau\end{cases}
\end{gathered}
$$

sound

$\operatorname{as}^{[5]}$

Where $v[n]$ is an additive sensor noise component, $a \in R$ is an attenuation factor, $s[n]$ is the original source signal, $\tau$ denotes the time at the sensor notices the acoustic event.

\subsection{Signal Distribution}

According to the model in Eq.(1), $x[n]=v[n]$ when $n \leq \tau$, i.e. the samples before the acoustic event belong exclusively to the noise component. We assume that it follows a normal distribution with zero mean and variance $E\left[v^{2}[n]\right]=\sigma_{0}^{2}$ :

$$
p\left(x[n], \theta_{0}\right) \sim N\left(0, \sigma_{0}^{2}\right)=\frac{1}{\sqrt{2 \pi} \sigma_{0}} e^{-\frac{x^{2}[n]}{2 \sigma_{0}^{2}}}
$$

Where $\theta_{0}=\sigma_{0}^{2}$, the Eq.(2) is the parameter modeling the Probability Density Function (PDF) before $\tau$.

In this paper, we assume very small time samples also follow Gaussian distribution.

As a result, a Gaussian PDF is selected as a model for the providing a signal samples after $\tau$, having the advantage of providing a more simple solution under this environment.

Then, the PDF after $\tau$ is expressed as:

$$
p\left(x[n], \theta_{1}\right) \sim N\left(0, \sigma_{1}^{2}\right)=\frac{1}{\sqrt{2 \pi} \sigma_{1}} e^{-\frac{x^{2}[n]}{2 \sigma_{1}^{2}}}
$$

Where $\theta_{1}=\sigma_{1}^{2}$ is the variance modeling the PDF after $\tau$.

\subsection{Statistical Framework}

Each node capture the samples can be modeled as a discrete random signal $x[n]$, with independent and identically distributed signal. Each signal's PDF is given by $p(x[n], \theta)$, where $\theta=\theta_{0}$ before the event at $n=\tau$ and $\theta=\theta_{1}$ when $n \geq \tau$. Thus, we assume:

$\hbar_{1}: \theta=\theta_{1}$, Node has capture the signal of sound source, sound event produced.

$\hbar_{0}: \theta=\theta_{0}$, Node has not capture the signal of sound source, no sound event produced.

The PDF of the signal $x[n]$ observed between the initial sample $x[n]$ and the current ample $x[k]$ can take two forms depending on the above hypotheses. Under the 'no event' hypothesis $\hbar_{0}$, the PDF is:

$$
p_{x \mid \hbar_{0}}=\prod_{n=0}^{K} p\left(x[n], \theta_{0}\right)
$$

On the other hand, under the 'event' hypothesis $\hbar_{1}$, the PDF would be:

$$
p_{x \mid \hbar_{1}}=\prod_{n=0}^{\tau-1} p\left(x[n], \theta_{0}\right) \prod_{n=\tau}^{K} p\left(x[n], \theta_{1}\right)
$$

The log-likelihood ratio (LLR) ${ }^{[6]}$ test is useful to decide between the two hypotheses. The LLR is defined by:

$$
\Lambda x \triangleq \ln \left(\frac{p_{x \mid \hbar_{1}}}{p_{x \mid \hbar_{0}}}\right)
$$

If user defined a threshold $\gamma$, the hypothesis $\hbar_{1}$ is decide if $\Lambda x>\gamma$, on the other hand, if $\Lambda x>\gamma$, the decision is $\hbar_{0}$.The Eq.(6) taking into account Eq.(4) and Eq.(5) become:

$$
\Lambda x[k, \tau]=\sum_{n=\tau}^{k} \ln \left(\frac{p\left(x[n], \theta_{1}\right)}{p\left(x[n], \theta_{0}\right)}\right)
$$


Depend on the unknown parameter $\theta_{0}, \theta_{1}$ and $\tau$, it is not possible to calculate the above quantity. But a generalized log-likelihood ratio (GLLR) can be defined by taking the place of the unknowns by their ML estimates ${ }^{[7]}$ :

$$
\begin{aligned}
\Gamma x[k] \triangleq \max _{1 \leq \tau \leq k} \Lambda x[k, \tau] \\
=\max _{1 \leq \tau \leq k} \sum_{n=\tau}^{k} \ln \left(\frac{p\left(x[n], \hat{\theta}_{0}\right)}{p\left(x[n], \hat{\theta}_{1}\right)}\right)
\end{aligned}
$$

Where $\hat{\theta}_{0}$ and $\hat{\theta}_{1}$ are the ML estimates of parameter. So if $\Gamma x[k]>\gamma$, it can select $\hbar_{1}$.

The next step is to estimate $\tau$ from the measured samples $x[0], \ldots, x[k]$.Using the value maximizing the likelihood $p_{x \mid \hbar_{1}}[k, \tau]$ to make the ML estimate for $\tau$ :

$$
\begin{aligned}
\hat{\tau} & \triangleq \underset{1 \leq \tau \leq k}{\operatorname{argmax}} p_{x \mid \hbar_{1}}[k, \tau] \\
& =\underset{1 \leq \tau \leq k}{\operatorname{argmax}} \Lambda x[k, \tau] \\
& =\underset{1 \leq \tau \leq k}{\operatorname{argmax}} \sum_{n=\tau}^{k} \ln \left(\frac{p\left(x[n], \hat{\theta}_{1}\right)}{p\left(x[n], \hat{\theta}_{0}\right)}\right)
\end{aligned}
$$

\section{Arrival Time Estimation}

\subsection{Review of Cumulative Sum Algorithm}

We define the instantaneous LLR at time $n$ as:

$$
l[n] \triangleq \Lambda x[n, n]=\ln \left(\frac{p\left(x[n], \theta_{1}\right)}{p\left(x[n], \theta_{0}\right)}\right)
$$

Cumulative Sum (CUSUM) the samples from 0 to $k$ as:

$$
s[k]=\sum_{n=0}^{k} l[n]
$$

From Eq.(10) and Eq.(11),the Eq.(6) can be expressed as:

$$
\Lambda x[k, \tau]=s[k]-s[\tau-1]
$$

Consider the PDF of samples, take Eq.(2) and Eq.(2) into Eq.(10):

$$
l[n]=\frac{1}{2}\left(\frac{1}{\sigma_{0}^{2}}-\frac{1}{\sigma_{1}^{2}}\right) x^{2}[n]+\frac{1}{2} \ln \left(\frac{\sigma_{0}^{2}}{\sigma_{1}^{2}}\right)
$$

Eq.(8) and Eq.(9) can be simply calculated in a recursive manner as:

$$
\begin{gathered}
\Gamma x[k]=s[k]-\min _{1 \leq \tau \leq k} s[\tau-1] \\
\hat{\tau}=\underset{1 \leq \tau \leq k}{\operatorname{argmin}} s[\tau-1]
\end{gathered}
$$

The CUSUM can be simply calculated as:

$$
s[k]=s[k-1]+l[k]
$$

Because the $\Gamma x[k]$ is compared to a positive threshold $\gamma$, so the Eq.(14) can be rewritten as:

where $\{z\}^{+} \triangleq \max (z, 0)$

$$
\Gamma x[k]=\{\Gamma x[k-1]+l[k]\}^{+}
$$

We assume that node has captured $k$ samples, and arrival time is included in these, i.e. the event must happen. So we can make ML estimate from $\tau=1$ to $\tau=K-1$, the ML estimate of $\tau$ is:

$$
\hat{\tau}=\underset{1 \leq \tau \leq K-1}{\operatorname{argmax}} \sum_{n=\tau}^{K-1} \ln \left(\frac{p\left(x[n], \hat{\theta}_{1}\right)}{p\left(x[n], \hat{\theta}_{0}\right)}\right)
$$

The Eq.(18) can simple expressed in CUSUM as :

$$
\hat{\tau}=\underset{1<\tau<K-1}{\operatorname{argmin}} s[\tau-1]
$$




\subsection{Parameter Estimates}

From the above known, we should know the variances $\sigma_{0}^{2}$ and $\sigma_{1}^{2}$ to calculate the ML estimate value of $\tau$, these parameters can be obtained from the observed data. The parameters $\sigma_{0}^{2}$ is estimated from the samples $x[0], \ldots, x\left[\tau^{\prime}-1\right]$, while the parameter $\sigma_{1}^{2}$ is estimated from the samples $x\left[\tau^{\prime}\right], \ldots, x[k]$ :

$$
\begin{aligned}
& \hat{\theta}_{0}^{(M L)}\left[\tau^{\prime}\right]=\hat{\sigma}_{0}^{2}\left[\tau^{\prime}\right]=\frac{1}{\tau^{\prime}} \sum_{n=0}^{\tau^{\prime}-1} x^{2}[n] \\
& \hat{\theta}_{1}^{(M L)}\left[\tau^{\prime}\right]=\hat{\sigma}_{1}^{2}\left[\tau^{\prime}\right]=\frac{1}{K-\tau^{\prime}} \sum_{n=\tau^{\prime}}^{K-1} x^{2}[n]
\end{aligned}
$$

where $k_{0} \leq \tau^{\prime} \leq k_{1}, \tau^{\prime}$ was the sample time and we assume that the event was produced between $k_{0}$ and $k_{1}$, so we can estimate the instantaneous ML estimation values of $\sigma_{0}^{2}$ and $\sigma_{1}^{2}$

$$
\begin{aligned}
\hat{\sigma}_{0}^{2} & =\frac{1}{k} x_{b}^{2}[n] \\
\hat{\sigma}_{1}^{2} & =\frac{1}{K-k} x_{b}^{2}[n]
\end{aligned}
$$

Where $K$ is the all sample time, $k$ is the time between $k_{0}$ and $k_{1}, x_{b}[n]$ is the summation of the sample before $n$.

From above, the node need to operate the algorithm for every $\tau^{\prime}$, increase the amount of calculation, and if the estimation of the statistics at the beginning and at the end of the observed signals, it will make the estimation not accurate. In order to overcome this disadvantage, we propose the CUSUM-FT (fixed parameters) to estimate parameters.

CUSUM-FT means determine a constant between $k_{0}$ and $k_{1}$, i.e. $k_{0} \leq T_{0} \leq k_{1}$, under this condition, the estimates are given by:

$$
\begin{aligned}
& \hat{\theta}_{0}^{\left(T_{0}\right)}=\hat{\sigma}_{0}^{2}=\frac{1}{T_{0}} \sum_{n=0}^{T_{0}-1} x^{2}[n] \\
& \hat{\theta}_{1}^{\left(T_{0}\right)}=\hat{\sigma}_{1}^{2}=\frac{1}{T_{0}} \sum_{n=K-T_{0}}^{K-1} x^{2}[n]
\end{aligned}
$$

Where the absolute difference between $T_{0}$ and arrival time estimation of ML is less than $\xi$,the test determine $\xi=20 \%$.

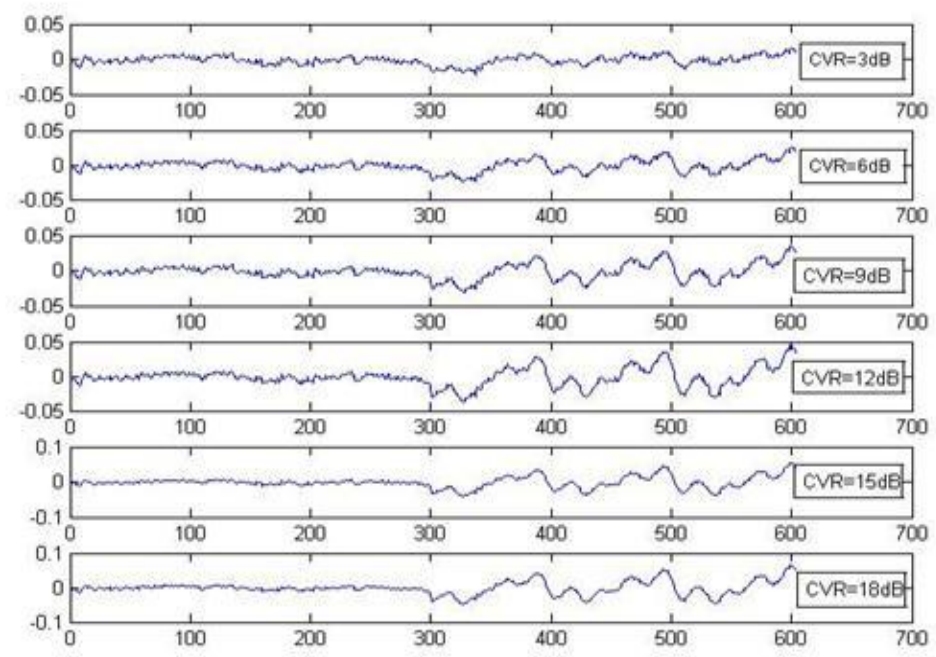

Figure 1. Sampling of Voice Signal 


\subsection{Discussion}

Let us define the change variance ratio $(\mathrm{CVR})^{[8]}$ as:

$$
C V R=10 \log _{10}\left(\sigma_{1}^{2} / \sigma_{0}^{2}\right)
$$

CVR is a another way of signal-to-noise ratio(SNR).

By adjusting the distance between the microphone and the sound source to collect different CVR signals. The waveform of signal as Figure 1. We analysis signal under different $C V R \in\{18,15,12,9,6,3\}$, the sampling time is 600 , and the event is produced in half of sampling time. i.e. it happened in $\tau=301$.Figure 2 and 3 is the cumulative sum curves(CSC) by these two algorithm. While the performance of both methods is very similar for CVR values above $6 \mathrm{~dB}$, the fixed parameters provide better accuracy than ML at low CVRs

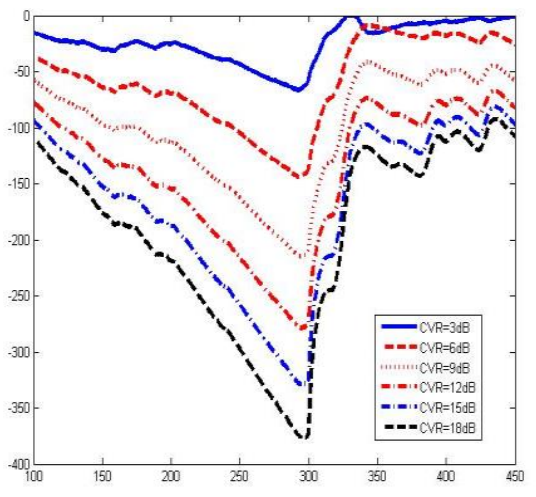

Figure 2. CSC of CUSUM-ML

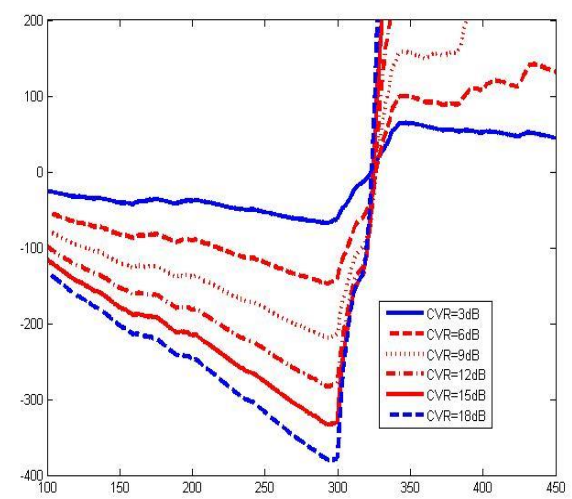

Figure 3. CSC of CUSUM-FT

In table 1 ,we can see the computational complexity of both algorithms. where we can see that ,while both have linear complexity. CUSUM-FT saves a considerable amount of operations.

Table 1. Computational Complexity

\begin{tabular}{|c|c|c|}
\hline Algorithm & Additions & Multiplications \\
\hline CUSUM-ML & $9 K-16 k_{0}-1$ & $13 K-28 k_{0}-1$ \\
\hline CUSUM-FT & $3 K+2 T_{0}+2$ & $2 K+2 T_{0}+9$ \\
\hline
\end{tabular}




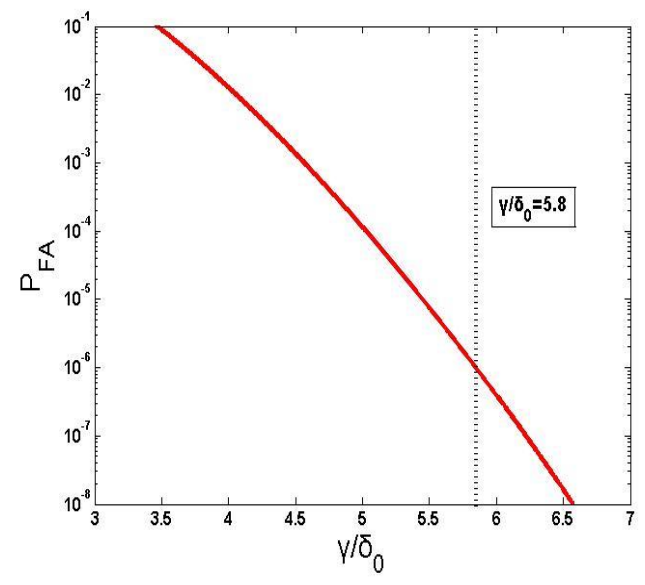

Figure 4. Effect of $\gamma / \sigma_{0}$ in Probability of False Alarm

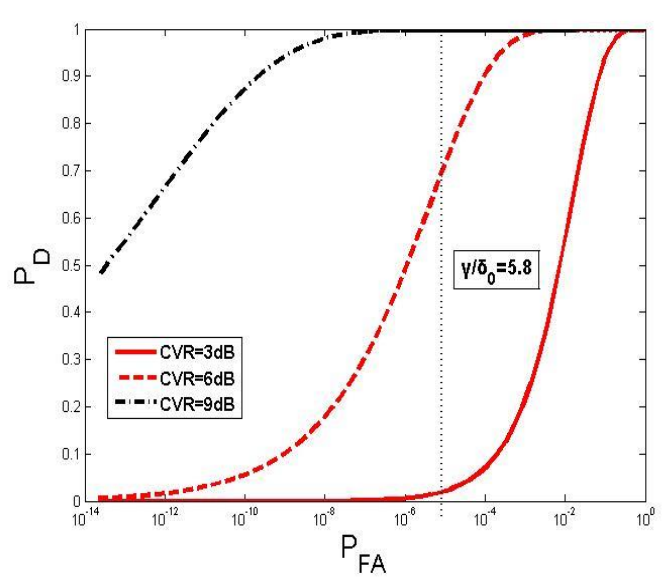

Figure 5. ROCC

\subsection{Threshold Selection}

In calibration step, selecting adequate threshold is carried out by studying the probability of observing high amplitude values given the noise power $\hat{\sigma}_{0}^{2}$. Since the nodes can only store a limited amount of samples, they must detect the event after observing. The probability of detection $P_{D}=1-\left(1-P_{\gamma \mid \hbar_{1}}\right)^{T}$, where $P_{\gamma \mid \hbar_{1}}$ is the probability of each sample taking a value higher than $\gamma$ under $\hbar_{1}$. Similarly, the probability of false alarm is given by $P_{F A}=1-\left(1-P_{\gamma \mid \hbar_{0}}\right)^{T} \cdot P_{\gamma \mid \hbar_{0}}$ and $P_{\gamma \mid \hbar_{1}}$ are:

$$
\begin{aligned}
& P_{\gamma \mid \hbar_{1}}=\operatorname{erfc}\left(\gamma / \sqrt{2 \sigma_{1}^{2}}\right) \\
& P_{\gamma \mid \hbar_{0}}=\operatorname{erfc}\left(\gamma / \sqrt{2 \sigma_{0}^{2}}\right)
\end{aligned}
$$

Where $\operatorname{erfc}()$ denotes the complementary error function ${ }^{[9]}$.Figure 4 show the probability of false alarm $P_{F A}$ given an amplitude threshold normalized with respect to the noise standard deviation, i.e. $\gamma / \sigma_{0}$.the corresponding receiver operating characteristic curves(ROCC) are shown in Figure 5 for different CVRs and $T=400$, .As shown in the Figure 4 and Figure 5 ,we select the threshold is $\gamma=5.84 \sigma_{0}$, it will make detecting an event with $P_{D} \approx 1$ when $C V R>7 \mathrm{~dB}$.

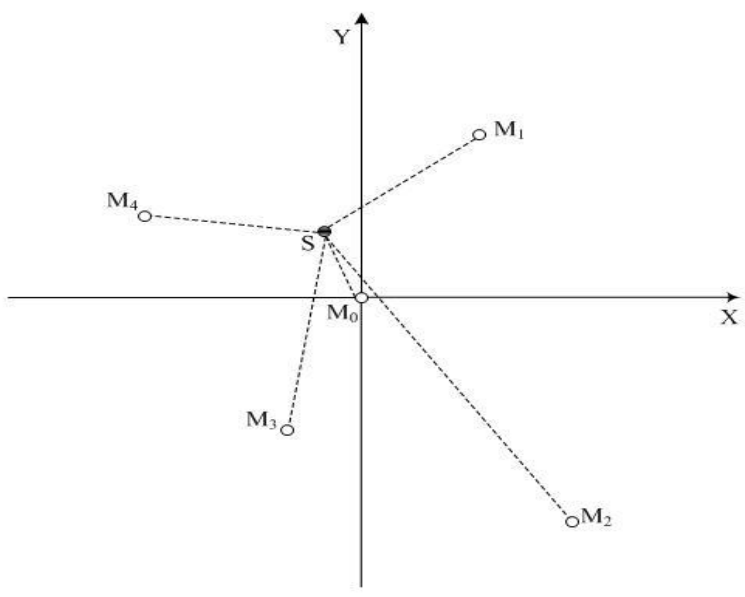

Figure 6. Array of Five Sensors 


\section{Sound Source Location and Organizational Process of System}

\subsection{The Principle of Sound Source Location}

We use a array of five sensors ${ }^{[10]}$ to locate the sound source. Constructed a Cartesian coordinate as Figure 6.The coordinate of each nodes is $(0,0),\left(x_{1}, y_{1}\right),\left(x_{2}, y_{2}\right),\left(x_{3}, y_{3}\right),\left(x_{4}, y_{4}\right)$. We assume $S$ is the sound source and its coordinate is $(x, y)$. The distance between $S$ and each nodes is $r_{0}, r_{1}, r_{2}, r_{3}, r_{4}$. The time of sound source arrive to $M_{0}$ is $t_{0}$. The time difference of $M_{i}$ and $M_{i}$ is $\tau_{i j}$.

From Figure 6 we can see that:

$$
\begin{gathered}
\left\{\begin{array}{c}
x^{2}+y^{2}=r_{0}^{2} \\
\left(x-x_{1}\right)^{2}+\left(y-y_{1}\right)^{2}=r_{1}^{2} \\
\left(x-x_{2}\right)^{2}+\left(y-y_{2}\right)^{2}=r_{2}^{2} \\
\left(x-x_{3}\right)^{2}+\left(y-y_{3}\right)^{2}=r_{3}^{2} \\
\left(x-x_{4}\right)^{2}+\left(y-y_{4}\right)^{2}=r_{4}^{2}
\end{array}\right. \\
\left\{\begin{array}{l}
r_{0}=c t_{0} \\
r_{1}=c\left(t_{0}+\tau_{10}\right) \\
r_{2}=c\left(t_{0}+\tau_{20}\right) \\
r_{3}=c\left(t_{0}+\tau_{30}\right) \\
r_{4}=c\left(t_{0}+\tau_{40}\right)
\end{array}\right.
\end{gathered}
$$

From Eq.29 we can know that the sound source coordinate is:

$$
\begin{gathered}
\left\{\begin{array}{l}
x=\frac{y_{2}\left(x_{1}^{2}+y_{1}^{2}+r_{1}^{2}-r_{1}^{2}\right)-y_{1}\left(x_{2}^{2}+y_{2}^{2}+r_{0}^{2}-r_{2}^{2}\right)}{2\left(x_{1} y_{2}-x_{2} y_{1}\right)} \\
y=\frac{x_{1}\left(x_{2}^{2}+y_{2}^{2}+r_{0}^{2}-r_{2}^{2}\right)-x_{2}\left(x_{1}^{2}+y_{1}^{2}+r_{0}^{2}-r_{1}^{2}\right)}{2\left(x_{1} y_{2}-x_{2} y_{1}\right)}
\end{array}\right. \\
\text { Get Eq.30 into Eq.31: } \\
x=\frac{y_{2}\left(x_{1}^{2}+y_{1}^{2}+2 c^{2} \tau_{10} t_{0}-c^{2} \tau_{10}^{2}\right)-y_{1}\left(x_{2}^{2}+y_{2}^{2}+c^{2} \tau_{20} t_{0}-c^{2} \tau_{20}^{2}\right)}{2\left(x_{1} y_{2}-x_{2} y_{1}\right)} \\
y=\frac{x_{1}\left(x_{2}^{2}+y_{2}^{2}+2 c^{2} \tau_{20} t_{0}-c^{2} \tau_{20}^{2}\right)-x_{2}\left(x_{1}^{2}+y_{1}^{2}+c^{2} \tau_{10} t_{0}-c^{2} \tau_{10}^{2}\right)}{2\left(x_{1} y_{2}-x_{2} y_{1}\right)}
\end{gathered}
$$

So we can use algorithm to calculate the time difference and cooperate with the speed of sound to locate sound source.

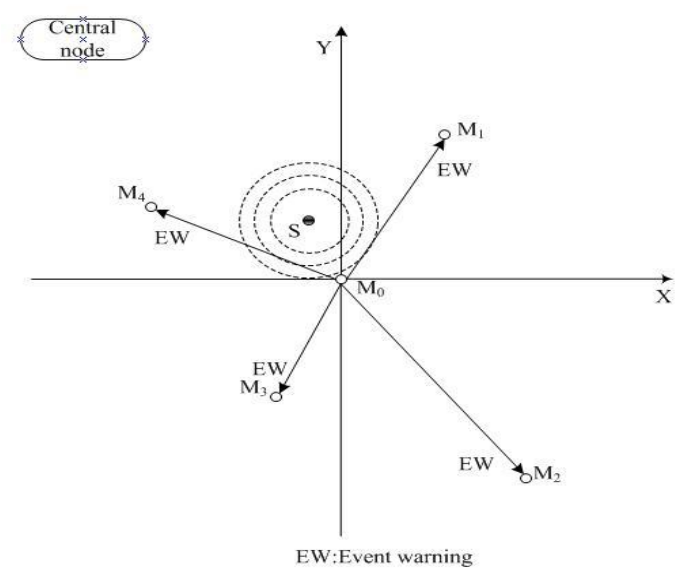

Figure 7. The Organize of Node (a)

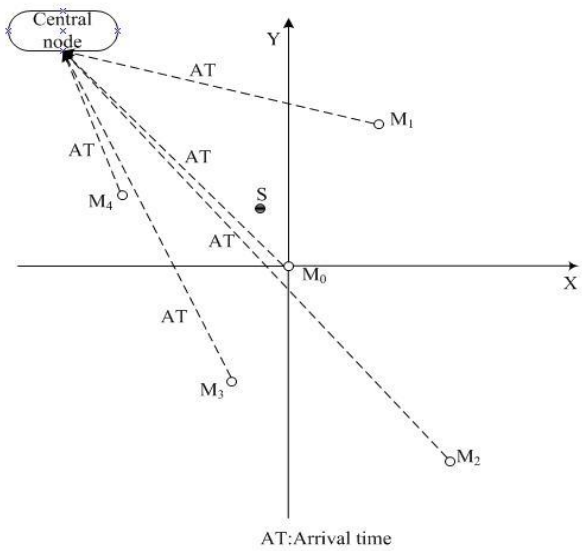

Figure 8. The Organize of Node (b) 


\subsection{The Principle of System Operation}

Before the sound event produced ,each node is in monitoring step, when the source make sound, the closest node detect the event produced, then run CUSUM-ML to calculate the arrival time and broadcast warning signals to sounding node. These node configure parameter to the node and run CUSUM-FT to calculate the time of sound arrive to each node, then these node send data about onset time of event to the sink, to locate the sound source. The Figure 7 and Figure 8 show that procedure.

\subsection{Error Analysis}

The algorithm has the deviation $\delta_{\tau}$, we assume $\delta_{\tau_{10}}=\delta_{\tau_{20}}=\delta_{\tau_{30}}=\delta_{\tau_{40}}=\delta_{\tau}$ and independent ${ }^{[11]}$,so the deviation in $x$ axis is :

$$
\delta_{x_{\tau}}=\delta_{\tau} \sqrt{\left(\frac{\partial x}{\partial \tau_{10}}\right)^{2}+\left(\frac{\partial x}{\partial \tau_{20}}\right)^{2}+\left(\frac{\partial x}{\partial t_{0}}\right)^{2}}
$$

Make the every parameters of Eq. 32 to partial derivative.

$$
\left\{\begin{array}{l}
\frac{\partial x}{\partial \tau_{10}}=\frac{-y_{2} c^{2}\left(t_{0}+\tau_{10}\right)}{x_{1} y_{2}-x_{2} y_{1}} \\
\frac{\partial x}{\partial \tau_{20}}=\frac{y_{1} c^{2}\left(t_{0}+2 \tau_{20}\right)}{2\left(x_{1} y_{2}-x_{2} y_{1}\right)} \\
\frac{\partial x}{\partial t_{0}}=\frac{c^{2}\left(y_{1} \tau_{20}-2 y_{2} \tau_{10}\right)}{2\left(x_{1} y_{2}-x_{2} y_{1}\right)}
\end{array}\right.
$$

Get Eq.(34) into Eq.(33), and we can also get the deviation in $y$ axis:

$$
\left\{\begin{array}{l}
\delta_{x_{\tau}}=\frac{c^{2} \delta_{\tau}}{2\left(x_{1} y_{2}-x_{2} y_{1}\right)} \sqrt{4 y_{2}^{2}\left(t_{0}+\tau_{10}\right)^{2}+y_{1}^{2}\left(t_{0}+2 \tau_{20}\right)^{2}+\left(y_{1} \tau_{20}-2 y_{2} \tau_{10}\right)^{2}} \\
\delta_{y_{\tau}}=\frac{c^{2} \delta_{\tau}}{2\left(x_{1} y_{2}-x_{2} y_{1}\right)} \sqrt{4 x_{1}^{2}\left(t_{0}+\tau_{20}\right)^{2}+x_{2}^{2}\left(t_{0}+2 \tau_{10}\right)^{2}+\left(x_{2} \tau_{10}-2 x_{1} \tau_{20}\right)^{2}}
\end{array}\right.
$$

So the accuracy of location is effected by $\delta_{\tau}$ and the distance between node and the sound

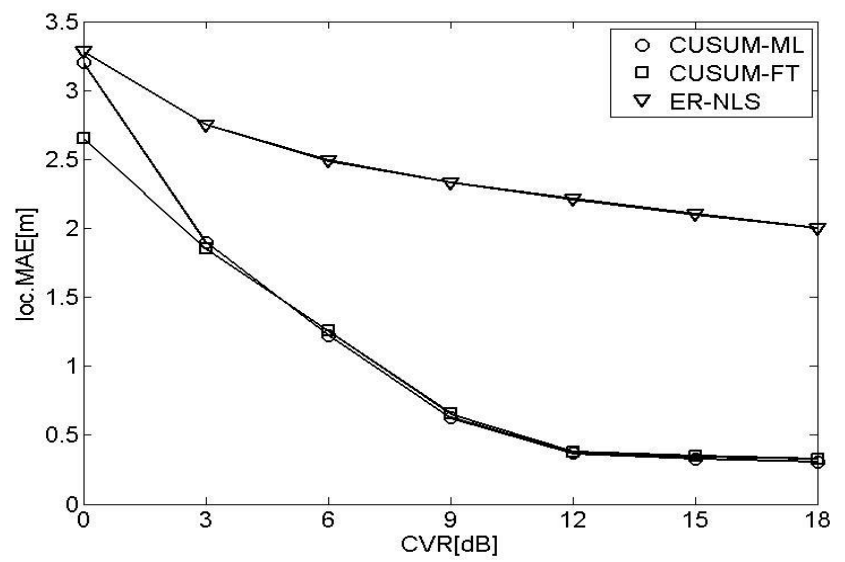

Figure 9. Mean Absolute Error of Two Algorithm 


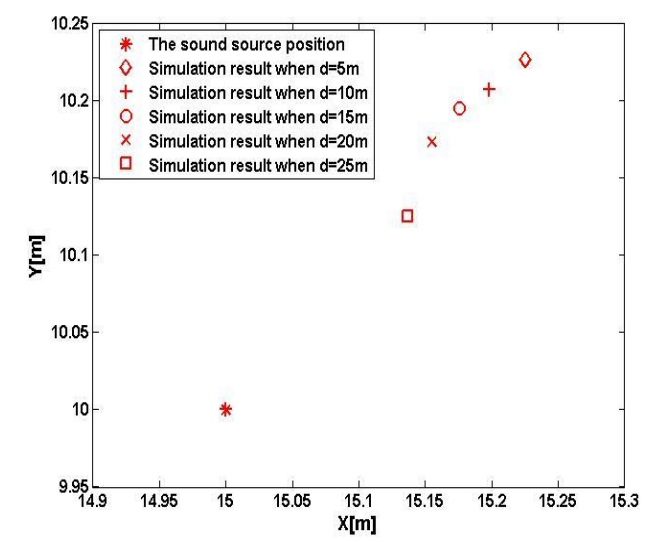

Figure 10. Result of First Aimulation

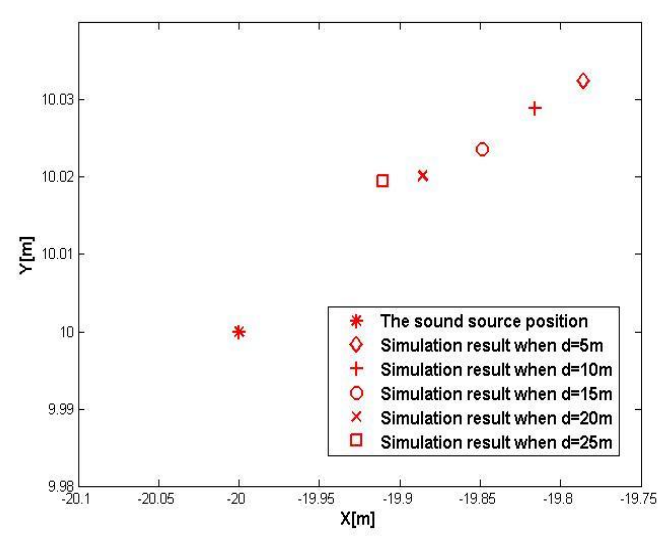

Figure 11. Result of Second Aimulation

\subsection{Emulation}

We assume the coordinate of these node is $(0,0),(d / 2,0),(0, d / 2),(-d / 2,0),(0,-d / 2)$ for $M_{0,} M_{1}, M_{2}, M_{3}, M_{4}$, we use MATLAB to programming . Taking sound source in $(0,0)$ and $d=10 \mathrm{~m}$.In this condition, measure accuracy of two algorithm under different CVR . Mean absolute error(MAE) can express this error precisely. MAE is express as:

$$
M A E=\frac{1}{N_{i} N_{j}} \sum_{i=1}^{N_{i}} \sum_{j=1}^{N_{j}} e_{i j}
$$

where $i$ and $j$ denote the number of node and results from the trial. The error $e_{i j}$ is defined as $e_{i j}=\left\|p_{s . i j}-\hat{p}_{s . i j}\right\|$,

The simulations were generated by using a sampling frequency ${ }^{[12]}$ of $f_{S}=16384 H_{z}$ and the amplitude threshold at each node is set as $\gamma=6 \hat{\sigma}_{0}$. As shown in Figure 9, we also comparing these algorithm with ER-NLS algorithm, which also used in the low cost location system.

We can see that these three algorithms has the same tendency. CUSUM-ML and CUSUM-FT provide better accuracy than ER-NLS, and CUSUM-FT has better location accuracy than ML at low CVRs.

In the case without signal attenuation and CVRs. Verify the accuracy affected by array of five sensors. Adjust the distance of node as $10 \mathrm{~m}, 20 \mathrm{~m}, 30 \mathrm{~m}, 40 \mathrm{~m}, 50 \mathrm{~m}$. Set the sound source in $S(15,10), S(-20,12)$,Figure 10 and Figure 11 are the result of two simulation. We can seen that the location accuracy is better along with the distance increases of the node.

But due to the distance of node increases, the space of node and the sound source also addition, so the signal attenuate more severe, and the formula as:

$$
L_{P}=L_{W}-O-D l m-A_{e}
$$

Where $L_{W}$ is the voice signal intensity emitted from the sound source, $K$ is the Standard attenuation, $O=10 \log _{10}(10,4 \pi)+20 \log _{10}(10, r)$ where $r$ is the distance, $D l m$ is the directivity factor, if it has reflective surface near the sound source, signal intensity need add $3 \mathrm{~dB}$, and the, $A_{e}$ is the additional factor. 


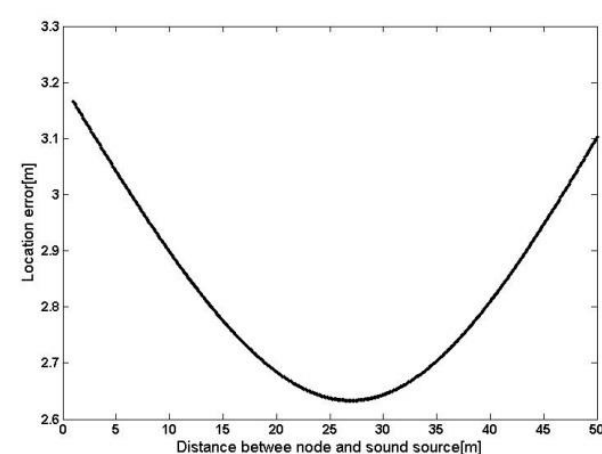

Figure 12. Relationship of Distance and LE

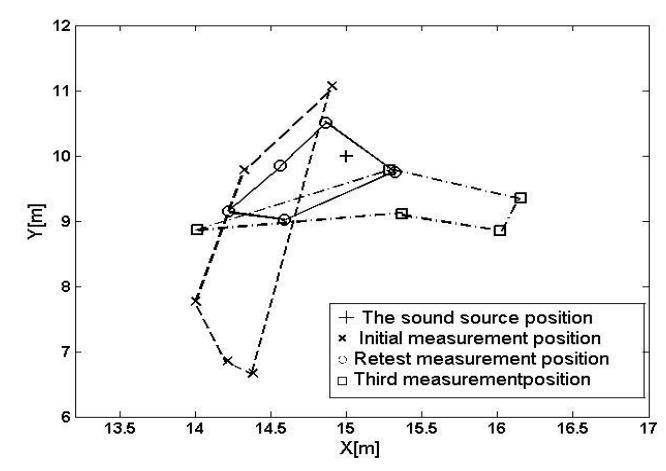

Figure 13. Result of each Measurement

Since signal attenuation, CVR of the node received signal has dropped, and the location accuracy is fall. This tendency of result is on the contrary with the result by the distance between node and the sound source.

After simulation, we find out the optimal distance of node and sound source. Figure 12 show the relation of distance and location error(LE) after consider signal attenuation.

From Figure 12 we can see that error tends to decline by increasing the distance at first, when the distance increasing to $d_{o p t}=26 \mathrm{~m}$ the error is least.

Then we present a retest method to reduce the location error. After sound source emitted the signal, the closest node monitored this signal first, soon after, it organize other four node estimate each arrival time and send the data to central node complete step one like above. Then the central node find out other four node space with the location result at step one the closest at $d_{\text {opt }}=26 \mathrm{~m}$, estimate arrival time and send data to central node location the sound source again at step two.Figure 13 show the simulation of many location. We deployed 50 node randomly at simulation, sound source coordinate is $(15,10)$, Figure 13 show the result of initial measurement, retest measurement and third measurement. We can see that location accuracy of retest measurement is improved, but the third measurement cannot improve the location accuracy further. So we only retest the sound source once.

\section{Conclusion}

We present algorithm based cumulative sum in this paper and also find the threshold of each node. By analyzing the algorithm by array of five sensors find out the factor of effect location accuracy, then we present the retest method to improve location accuracy. The simulation show that the algorithm and the way of organization node is effective.

\section{Acknowledgements}

Many people have made invaluable contributions, both directly and indirectly to my research. I would like to express my warmest gratitude to NAIHE for his instructive suggestions and valuable comments on the writing of this thesis. Without his invaluable help and generous encouragement, the present thesis would not have been accomplished.

I also wish to thank my colleagues Guilin University of Technology, who helped me search for reference. What's more, I wish to say "Thanks!" to the 100 subjects in this study for providing me chances to do the empirical study.

Last but not the least, express my warmest gratitude my family who have been assisting, supporting and caring for me all of my life. 


\section{References}

[1] X L Huang, F B Wang, W J Duan, Y Yang, "Error Analysis and Calibration of TDOA Ranging for Wireless Sensor Networks[J]”, Computer Measurement \& Control. 2008. vol. 16, no. 7, pp. 1053-1056

[2] Canclini A, Bestagini P, Antonacci F.A Robust and Low Complexity Source Localization Algorithm for Asynchronous Distributed Microphone Networks [J].IEEE/ACM TRANSACTIONS ON AUDIO SPEECH AND LANGUAGE PROCESSING, 2015, vol. 23, no. 10, pp. 1563-1575

[3] Cakir M. Accurate and Fast Reference Signal Estimation with Averaged-LMS for Active Power Filters[J].INTERNATIONAL REVIEW OF ELECTRICAL ENGINEERING IREE, 2012, vol. 7, no. 2, pp. 3739- 3748

[4] Segura Carlos, Hernando Javier.3D Joint Speaker Position and Orientation Tracking with Particle Filters[J].SENSORS, 2014, vol. 14, no. 2, pp. 2259-2279.

[5] ZHAO L. Speech Signal Processing[M].Machinery Industry Press,2009, pp. 24-27

[6] ZHANG W, et al. Automatic Digital Modulation Recognition Algorithms Based on approximately logarithm Likelihood Method [J]. Signal processing.2007, vol. 23, no. 1, pp. 58-63

[7] ZHI Y F, et al. Signal Processing Method Based on logarithm Likelihood[J].Journal of Northwestern Poly technical University. 2008, vol. 26, no. 2, pp. 179-183

[8] Maximo Cobos, Jaume Segura. Cumulative-Sum-Based Location of Sound Events in Low-Cost Wireless Acoustic Sensor NETWORK[J].IEEE/ACM TRANSACTIONS ON AUDIO,SPEECH AND LANGUAGE PROCESSING. 2014,22(12), pp. 1792-1802

[9] JIN H L, et al. The Necessary and Sufficient Condition of the Families of Bent Complementary Function Pairs[J].Journal of Electronics \& Information Technology.2008, vol. 30, no. 6,pp. 1397-1399

[10] LIU Y R, et al. Research of Passive Acoustic Location Based on Rectangular Pyramid Array of Six Sensors[J].Chinese Journal of SENSORS AND ACTUATORS.2014, vol. 27, no. 2, pp. 252-256

[11] LIN X D, et al. Passive acoustic location algorithm based on a six-element detection array and its performance analysis[J]. Technical Acoustics.2008, vol. 27, no. 2,pp. 192-196

[12] MAO J L, et al. Optimization algorithm for sampling frequency assignment in wireless sensor networkbased control system[J]. Control and Decision.2007, vol. 22, no. 1, pp. 45-48

\section{Authors}

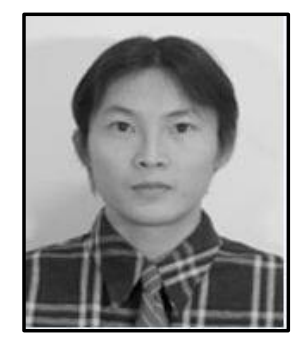

SHEN Xian-hao. Ph. D., Guilin University of Technology, NO.12 Jiangan Road, Guilin, Guangxi Province, China. (541004) Tel:15077301839, E-mail: 25337698@qq.com

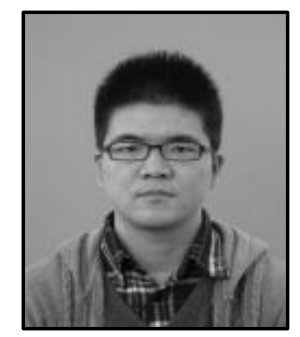

NAI He. Postgraduate, Guilin University of Technology, NO.12 Jiangan Road, Guilin, Guangxi Province, China. (541004) Tel:18290065616, E-mail:985515263@qq.com 
International Journal of Grid and Distributed Computing

Vol. 9, No. 6 (2016) 